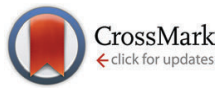

Cite this: DOI: 10.1039/c5sm00942a

\title{
A molecular dynamics study of bond exchange reactions in covalent adaptable networks $\dagger$
}

\author{
Hua Yang, ${ }^{\text {ab }}$ Kai Yu, ${ }^{a}$ Xiaoming $\mathrm{Mu}^{a}{ }^{a}$ Xinghua Shi, ${ }^{\mathrm{c}}$ Yujie Wei, ${ }^{\mathrm{c}}$ Yafang Guo ${ }^{\mathrm{b}}$ and \\ H. Jerry Qi*a
}

Covalent adaptable networks are polymers that can alter the arrangement of network connections by bond exchange reactions where an active unit attaches to an existing bond then kicks off its pre-existing peer to form a new bond. When the polymer is stretched, bond exchange reactions lead to stress relaxation and plastic deformation, or the so-called reforming. In addition, two pieces of polymers can be rejoined together without introducing additional monomers or chemicals on the interface, enabling welding and reprocessing. Although covalent adaptable networks have been researched extensively in the past, knowledge about the macromolecular level network alternations is limited. In this study, molecular dynamics simulations are used to investigate the macromolecular details of bond exchange reactions in a recently reported epoxy system. An algorithm for bond exchange reactions is first developed and applied to study a crosslinking network formed by epoxy resin DGEBA with the crosslinking agent tricarballylic acid. The trace of the active units is tracked to show the migration of these units within the network. Network properties, such as the distance between two neighboring crosslink sites, the chain angle, and the initial modulus, are examined after each iteration of the bond exchange reactions to provide detailed information about how material behaviors and macromolecular structure evolve. Stress relaxation simulations are also conducted. It is found that even though bond exchange reactions change the macroscopic shape of the network, microscopic network characteristic features, such as the distance between two neighboring crosslink sites and the chain angle, relax back to the unstretched isotropic state. Comparison with a recent scaling theory also shows good agreement.

Received 21st April 2015, Accepted 25th June 2015

DOI: $10.1039 / c 5 s m 00942 a$

www.rsc.org/softmatter
A typical CAN alternates its network through an additionfragmentation process, as illustrated in Fig. 1. An active unit attaches to an existing bond first (Fig. 1a); the newly formed tertiary structure is not stable, leading to one of the units being kicked-off, resulting in a new bond and an active unit (Fig. 1b and c). Such a reaction is often called a bond exchange reaction (BER). At the network level, BERs allow the network to be rearranged, ${ }^{7,8}$ which leads to many interesting behaviors. ${ }^{9,10}$ For example, Scott et al. ${ }^{11}$ demonstrated that light could trigger radical induced BERs for stress relaxation and plastic deformation. This material was later explored for a variety of applications, such as flaw retardation, surface patterning, foldable structures. ${ }^{12-16}$ In Scott et al., radicals, which are produced from photolysis of photoinitiators, are required to maintain the BERs. Once photoinitiators are depleted, the BERs stop. Recently, Leibler's group proposed an epoxy-based CAN where the BERs are catalyzed and controlled by temperature, which can be used for thermoforming, welding, and powder-based reprocessing. ${ }^{17,18}$

As a new group of active polymers, CANs have drawn significant interest in recent years, including new material systems developed by Lu et al. ${ }^{19}$ and Taynton et al. ${ }^{20}$ Theoretical studies were conducted in the past. For example, Leibler and coworkers ${ }^{21}$ 


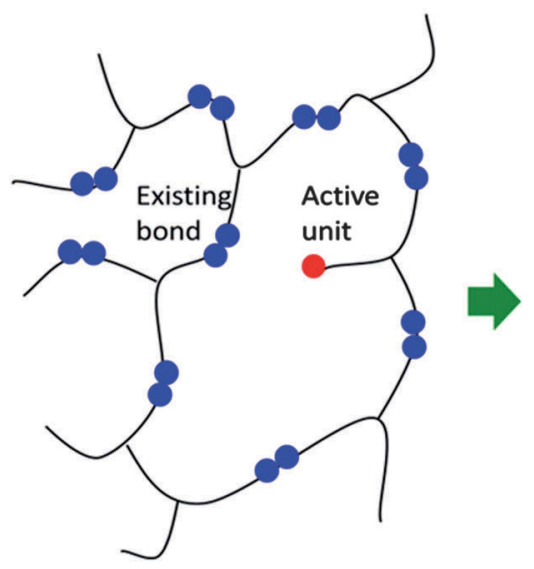

(a)

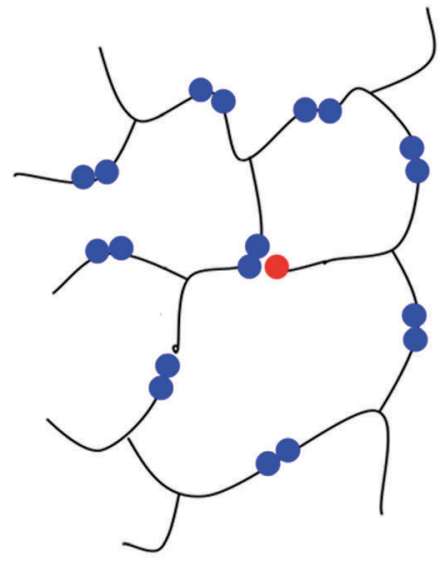

(b)

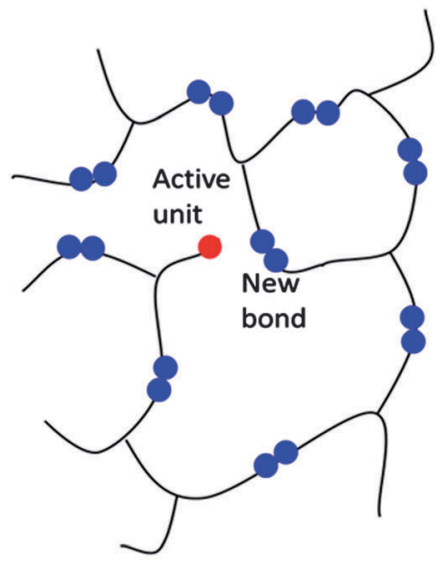

(c)

Fig. 1 Schematic graphs of a bond exchange reaction: (a) before exchange; (b) intermediate state; (c) after exchange.

studied the diffusivity and stress relaxation in a reversible network by considering the reptation of stickers, which are responsible for reversible crosslinks. Rubinstein and Semenov further studied the dynamics of thermoreversible gelation of associating polymer solutions. ${ }^{22-24}$ Recently, Long et al. ${ }^{15,25}$ Long et al.,${ }^{5,26}$ and Ma et $a l^{27}$ studied the stress relaxation due to dynamic bonds in different material systems at the continuum mechanics level. Stukhalin $e t ~ a l^{28}$ developed a scaling theory to study the selfhealing process by forming a reversible bond with each other in hybrid reversible/permanent networks. Although these studies greatly advanced our understanding of these materials, it will be very helpful if we can delineate the macromolecular level details of network rearrangement by BERs. For example, one fundamental question is if the BER-induced plastic deformation would change the structure of the network. Smallenburg et al. ${ }^{29}$ used the patchy particle model to study the swelling behaviors of reversible networks and found an entropy-driven phase separation. Molecular dynamics (MD) simulations ${ }^{30}$ are a well-established and important tool that allows practical problems to be explored in detail at macromolecular scales, which are difficult to extract directly from macroscopic experiments. For example, Yoshioka et al. ${ }^{31}$ used MD simulations to examine glass transition temperature $\left(T_{\mathrm{g}}\right)$ of the freeze-dried formulation containing polymer excipients. Li and Strachan $^{32}$ performed extensive characterization of the thermomechanical response of epoxy EPON862 with curing agent DETDA using MD simulations. Gou et al. ${ }^{33}$ also conducted MD simulations to investigate the interface bonding of single-walled carbon nanotube reinforced epoxy composites. Bermejo and Ugarte study the crosslinking process of PVA. ${ }^{34}$ For BERs, Rottach et al. ${ }^{35}$ used the coarse-grained approach to investigate stress relaxation of sequential crosslinking and scission of polymer networks, which focused mainly on stress relaxation. Bandyopadhyay ${ }^{36}$ proposed an MD method to simulate the sequence of bond formation and breakage with the goal of building an accurate network. In this work, we study the rearrangement of a polymer network in the epoxy developed by Montarnal, ${ }^{17}$ where BERs are achieved through transesterification reactions. In this material, since the BERs are catalyzed and can continue as long as the temperature is high, it is reasonable not to consider the termination. The goal of this paper is to develop a full atomistic MD simulation procedure for BERs to study the evolution of a polymer network. With the MD simulations, we can track the migration of active atoms during a relay of BERs. We also inspect several network properties, including the distance between two neighboring crosslink sites, the chain angles, initial modulus, stress relaxation, and shape reforming etc., for any changes in the network during BERs. Finally, MD simulation of stress relaxation is compared with the existing theoretical studies.

\section{Simulation details}

The MD model for the thermosetting epoxy network polymer with exchangeable bonds used in this study was prepared by following Montarnal's work. ${ }^{37}$ The initial system consists of un-crosslinked epoxy resin DGEBA and crosslinking agent tricarballylic acid. The molecular structures are shown in Fig. 2a, where a DGEBA has two active sites and a tricarballylic acid has three active sites. In order to maintain the 1:1 ratio among active sites in epoxy monomers and curing agent monomers, a mixture of 90 DGEBA molecules and 60 tricarballylic acid molecules, which result in a total atom number of 5430, was built using an amorphous cell module in Materials Studio ${ }^{38}$ with charges assigned using the group charge method. All the simulations were performed using the PCFF forcefield $^{39}$ and LAMMPS molecular dynamics software ${ }^{40}$ as provided by Sandia National Laboratories. The Nose-Hoover thermostat and barostat ${ }^{41,42}$ were used for temperature and pressure control, respectively. 3-D periodic boundary conditions were employed for all simulations to remove possible surface effects.

\subsection{Crosslinking process}

The first step in studying the BERs is to build a crosslinking network. The MD process of crosslinking epoxy resin compounds was investigated by many researchers and typically includes 
<smiles>CC(C)(c1ccc(OCC2CO2)cc1)c1ccc(OCC2CO2)cc1</smiles>

(a)<smiles>CC(C)(c1ccc(OCC(O)c2ccccc2)cc1)c1ccc(OCC(O)C2CCCC2)cc1</smiles>

(b)

Fig. 2 (a) Molecular structure of tricarballylic acid and DGEBA. (b) Activation of tricarballylic acid and DGEBA ends for the crosslink. The atoms in the red circle showed their activity toward the crosslinking reaction.

two parts: the first part is a connection where monomers are connected by following a certain pre-defined criterion; the second part is relaxation where the artificial energy due to the connection part is relaxed. In addition, in order to avoid high artificial energy build-up in the connection part, an iterative approach is typically used to limit the number of connection reactions in one iteration. For example, in Xu and Wu's work, ${ }^{43}$ only one connection reaction was allowed in each iteration, followed by 1000 time steps of MD relaxation of the newly formed topology. Apparently, this protocol requires a significant amount of computational time. Varshney et al. ${ }^{44}$ combined Heine's dynamic crosslinking concept ${ }^{45}$ by using a cutoff distance with Wu and Xu's iterative energy minimization method. Specifically, they connected all the atom pairs that meet the cutoff distance criterion. This would lead to a very dramatic system energy change if too many pairs were connected. Therefore, they used a multistep relaxation procedure for relaxing the system by gradually increasing the force constant. Varshney et $a .^{44}$ also compared three different crosslinking approaches where different active pairs may have preferred or equal chances of connection. Their results indicated that there was no significant difference among the final crosslinked systems, except the computational time.

In this work, we combine $\mathrm{Wu}$ and Xu's and Varshney's works, i.e., we use Varshney's method in the connection procedure and $\mathrm{Wu}$ and $\mathrm{Xu}$ 's method in the relaxation procedure, which consists of one molecular mechanics (MM) energy minimization procedure followed by a MD relaxation. Fig. 3 shows the flowchart of the method for crosslinking, which consists of 7 steps. In Step 1, the initial system is equilibrated using $N V T$ and $N P T$ sequentially at the atmosphere pressure and $300 \mathrm{~K}$ for 100 ps. Once the initial system is equilibrated, all the potential reactive sites in both tricarballylic acid and DGEBA are activated. This is achieved by removing hydrogen atoms from tricarballylic acid's carboxyl groups to make it chemically active. In the case of DGEBA, removing one of the hydrogen

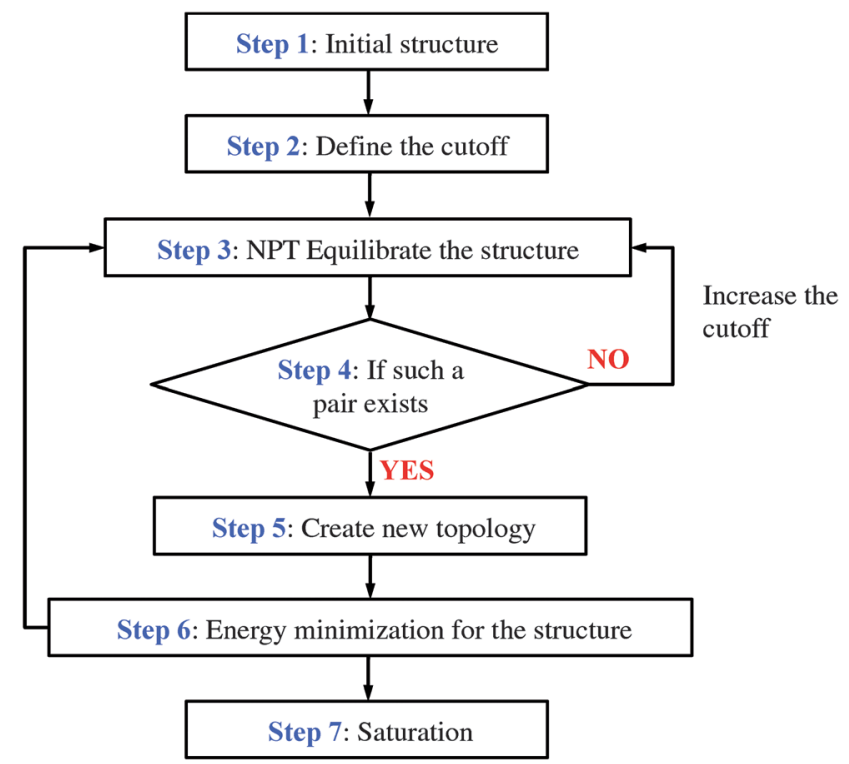

Fig. 3 Flowchart of the crosslink process.

atoms creates a reactive methylene end group. It should be noted that although all the potential reactive sites are chemically activated in this step, two active sites are connected only when their relative distance is within a cutoff distance. In addition, following the observations from Varshney et al., ${ }^{44}$ we do not assign priorities to any reactive sites. The reactive macromolecular segments with reactive sites will be used in the next step. The schematics of reactive macromolecular segments are shown in Fig. $2 b$.

After the initial preparation of the system in Step 1, Step 2 starts the iterative crosslinking process. In Step 2, a cutoff distance $(4 \AA)$ is defined. Step 3 conducts a NPT equilibrium for 100 ps. The distances between all active atom pairs are checked in Step 4. If no pairs have the distance less than the cut-off distance, it is increased by $0.25 \AA$ and Step 3 is repeated. 
If at least one pair is found within the cut-off distance, the topological information is updated by introducing new bonds (formed by two atoms in these pairs), angles, dihedrals and proper angles into the system in Step 5, which is then followed by MM energy minimization and 100 ps NPT relaxation in Step 6 to relieve any unfavorable interactions due to the formation of new bonds. Steps 3 to 6 are repeated until all the monomers are bonded together to form one network. Once the construction is completed, all non-hydrogen atoms in the system are saturated with hydrogen atoms in Step 7.

\subsection{Bond exchange reaction process}

Various kinds of reversible covalent chemistry can be used to produce CANs, such as the Diels-Alder (DA) reaction, ${ }^{46}$ the radical addition fragmentation chain transfer (RAFT) reaction ${ }^{47}$ and the transesterification reaction. ${ }^{17}$ In this work, the transesterification-type BER is used to enable the network rearrangement. The crosslinking process forms a network that contains ester and alcohol functional groups. The pendent alcohol and ester functional groups undergo a transesterification-type BER to enable the network rearrangement. The BER allowed in this system is illustrated in Fig. 4, where a transesterification reaction occurs between an ester and an alcohol functional group (marked in red square in Fig. 4a), resulting in a new ester functional group (red circle on the left in Fig. 4b) and an alcohol functional group with a released oxygen (red circle on the right). The latter becomes active to continue the BER.

The flowchart describing the BER algorithm using the MD method is shown in Fig. 5. In Step 1, all the potential reactive sites in the network are activated by removing hydrogen atoms from the alcohol groups and by changing the double bond into a single bond in the ester functional groups. In the epoxy system in Montarnal et al., ${ }^{17}$ BER is mediated by the presence of catalysts; we therefore assume that the catalysts are uniformly distributed. However, whether or not an active atom will be exchanged between two active sites depends on the relative distance, as well as the system energy after exchanging, which will be discussed in the followings.

The number of active oxygen atoms and active carbon atoms are both 180 . These reactive molecular segments with reactive sites of oxygen or carbon will be used in the BERs. In addition, we assume that the BER only occurs between an alcohol and an ester functional group by following the reaction scheme shown in Fig. 4. In Step 2, in order to avoid dramatic change in energy, a smaller initial cutoff distance $(3.3 \AA)$ is employed at the beginning of the simulation. The reactive system is then equilibrated in Step 3 by using the NVT ensemble for $100 \mathrm{ps}$ at $450 \mathrm{~K}$ to achieve a relaxed network. In Step 4, the distances between all active pairs (one active hydrogen and one active oxygen) are calculated. In Step 5, any active pairs whose distances are within the cutoff distance are connected by forming new bonds. If no such a pair exists, Steps 3 and 4 will be repeated until such a pair can be found. In Steps 6 and 7, the system energy is minimized then relaxed by using the $N V T$ ensemble. In Step 8, the bond energies between the newly formed bond and the initial bond are compared. If the newly formed bond energy is lower than the initial bond energy, the initial bond is broken in Step 9 and vice versa. In Step 10, the topology information is updated by introducing new bonds, angles, dihedrals and improper angles into the system. Another round of minimization and 100 ps

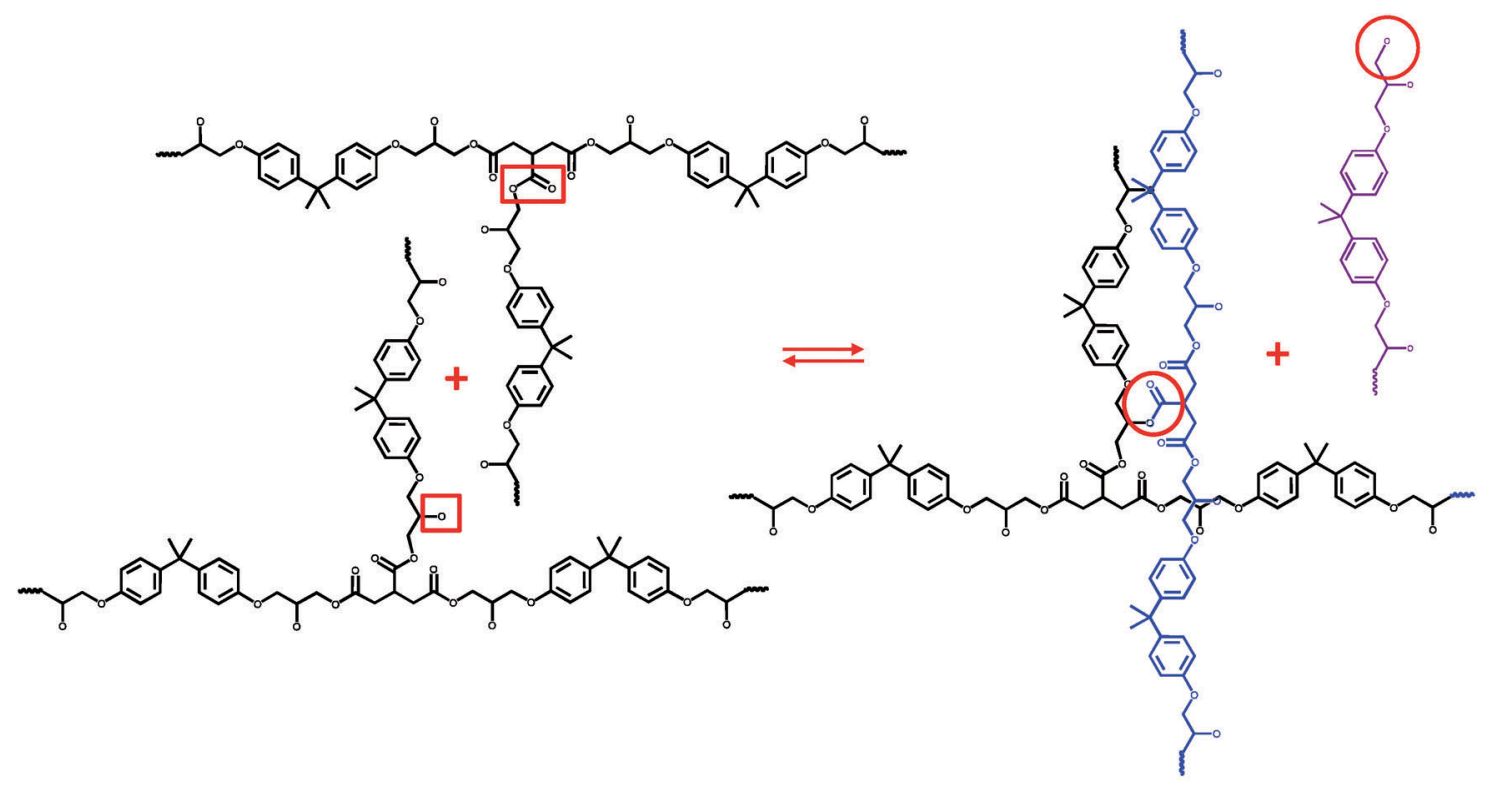

(a)

(b)

Fig. 4 Schematic of the bond exchange reaction (a) the transesterification reaction occurs between ester and alcohol functional groups, which are in the red squares (b) after the transesterification, a new ester functional group (red circle on the left) is formed, and the released oxygen (red circle on the right) which belongs to alcohol functional group becomes an active atom again. 


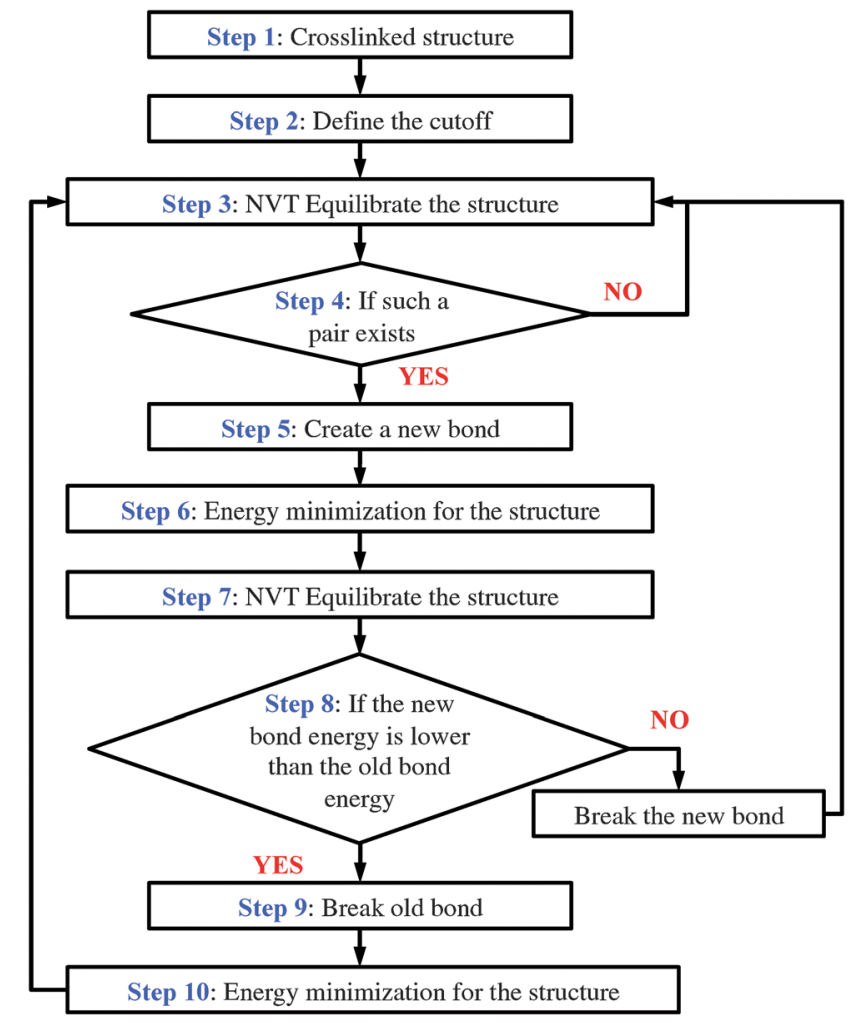

Fig. 5 Flowchart of the BER process.

NVT relaxation were performed to relieve any unfavorable interactions due to the formation of new bonds. Steps 3 to 10 are repeated for 100 iterations.

\section{Results and discussion}

\subsection{The crosslinked system}

The final crosslinked system is shown in Fig. 6. The zoomed-in graph in Fig. 6b confirms that the final network is an integrated structure. The glass transition temperature is determined to be $312 \mathrm{~K}$ for such a system (see $\mathrm{ESI} \dagger$ for details).

\subsection{Evolution of network properties}

The BER process described above is used to conduct MD simulations. Here, in order to obtain a large enough sampling pool, we allow 100 iterations of the BER process, which results in a total number of 605 BERs. Since there are 180 active oxygen atoms, all the active atoms participate in BER. The number of BERs in each iteration is shown in Fig. S2 in the ESI. $\dagger$ There is an average of $\sim 6$ BERs in each iteration.

With the results from above simulations, we investigate the topological and mechanical properties during the evolution of the network. We first track the trajectory of active atoms as they move around the macromolecular chains in the network. For topological properties, we monitor two important parameters: the distance between crosslinking sites and the chain angle. For mechanical properties, we check the initial modulus as BERs proceeding.

3.2.1 The trajectory of active atoms. In a BER, an active atom reacts with a functional group in the polymer chain, which subsequently generates a new bond and a new active atom that participates in the next BER. In such a way, a BER continues in a relay of active atoms. It is therefore interesting to track the trajectory of active atoms in this relay, which is shown in Fig. 7. The purple active atom (oxygen, purple color) at the end of a chain (cyan color) approaches a functional group (pink color in the green chain) in Fig. 7a, then reacts with it by forming a new connected chain (cyan color and green color) and a new chain (green color) with an active atom at the end in Fig. 7b. The released active atom (oxygen, active) then continues to search for another functional group (blue pair) in Fig. 7c and reacts with it as shown in Fig. 7d. Once the red atom forms a new bond with one blue atom (carbon), another blue atom (oxygen) is kicked off and becomes active again. This process continues. After 100 iterations of BER simulations, the relay goes through 11 BER reactions. The trajectory and its travel distance are shown in Fig. 8a and b. If more BER iterations are conducted, we expect that the trajectory of the relay would pervade the whole system, which is attributed to the fact that potential active atoms are randomly distributed within the system. Certainly, it would not

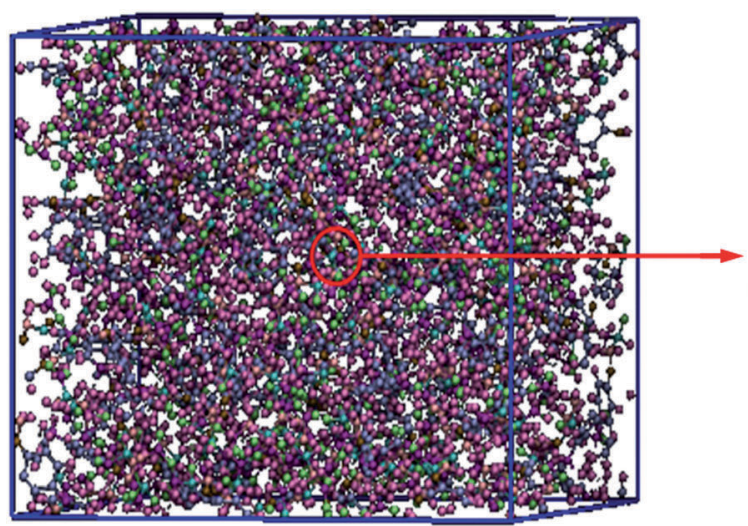

(a)

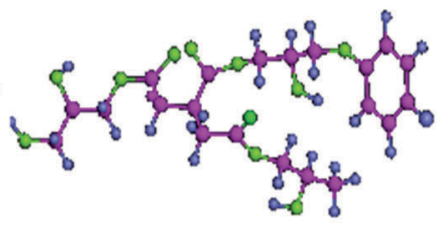

(b)

Fig. 6 (a) The crosslinked network; (b) the zoomed-in view of the network. Carbon (pink); oxygen (green); hydrogen (purple). 


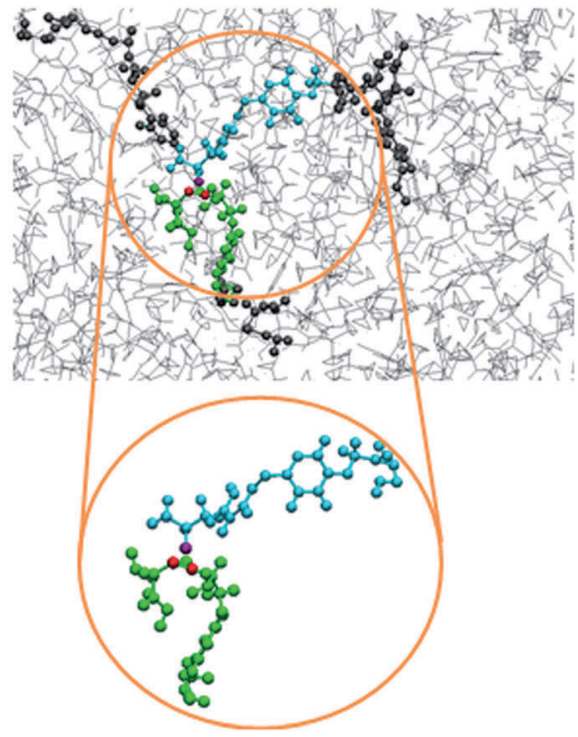

(a)

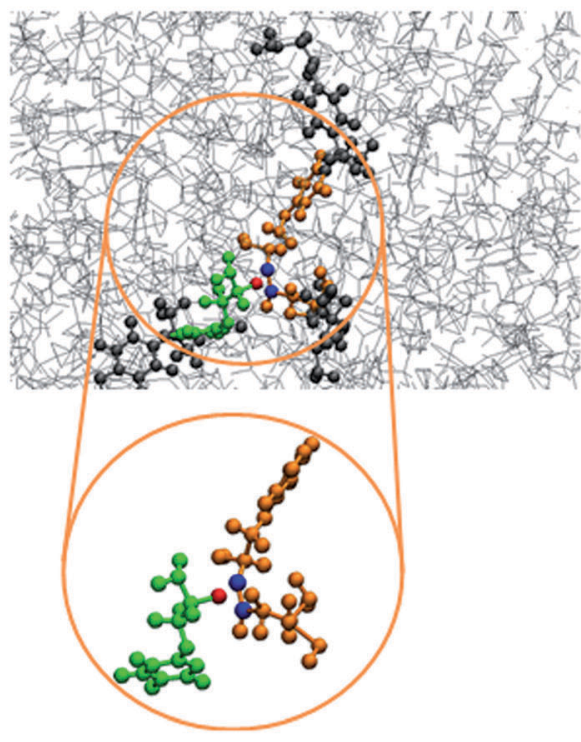

(c)

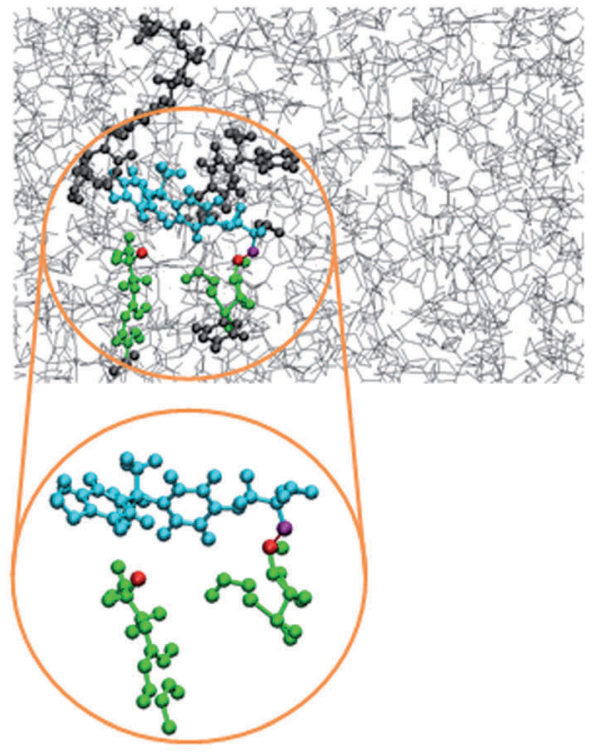

(b)

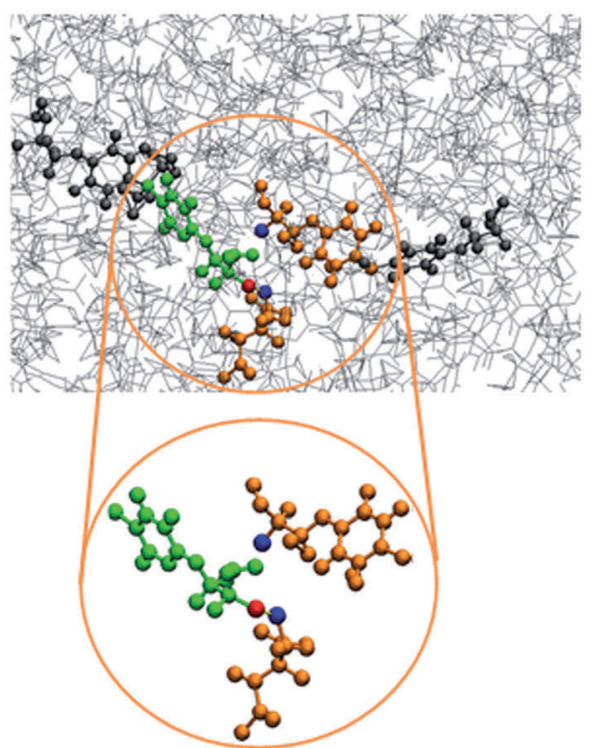

(d)

Fig. 7 The relay of active atoms in BERs: (a) the purple active atom meets a red atom pair; (b) the purple atom reacts with the red atom pair by creating a new bond with one red atom and releasing the other red atom; (c) the released red atom meets a blue atom pair; (d) the red atom reacts with the blue pair by forming a new bond with one blue atom and releasing the other blue atom.

be true if some termination mechanisms, such as the bimolecular termination mechanism in radical assisted BERs, are considered. Fig. 8c shows the average travel distance of all active atoms after 100 iterations of BERs, which are about 20 nanoseconds (ns) with $\sim 600$ BERs, showing clearly that with the BER process continues, the active atoms move further.

3.2.2 Evolution of network topological properties. To check the topological properties of the network, we use two parameters: the distance between crosslinking sites and the chain angle. The distance between two crosslinking sites is defined as the distance between two carbon atoms at the crosslinking sites, as shown in Fig. 9. The chain angle is the angle between the line connecting two crosslinking sites and one of the axes. Here, for the convenience, we use the $x$-axis, which is the stretching direction in the next section.

Fig. 10 shows the average values and their standard deviations of the distance between two crosslinking sites and the chain angle. It can be seen that BERs do not change these network characteristics. The average distance between two neighboring crosslinking sites remains to be $15.6 \AA$, and the chain angle is $58.8^{\circ}$.

The distributions of the distance between two neighboring crosslinking sites and the chain angle do not change during the BER process. Fig. S3 (in ESI $\dagger$ ) showed the distributions at the 


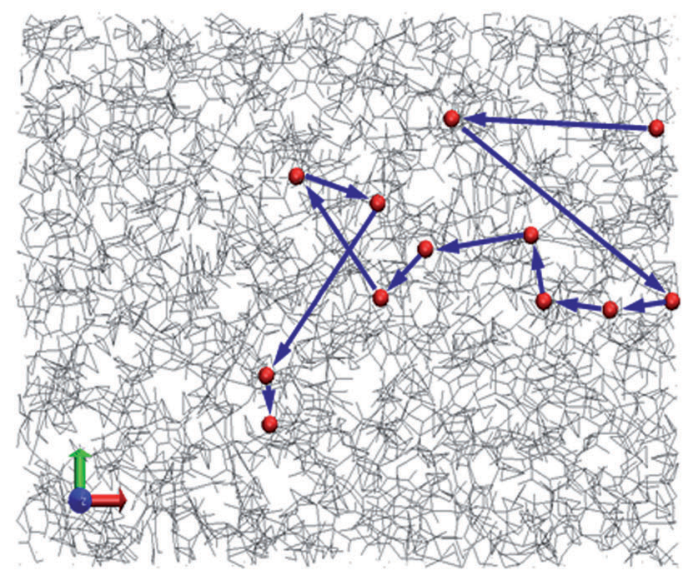

(a)

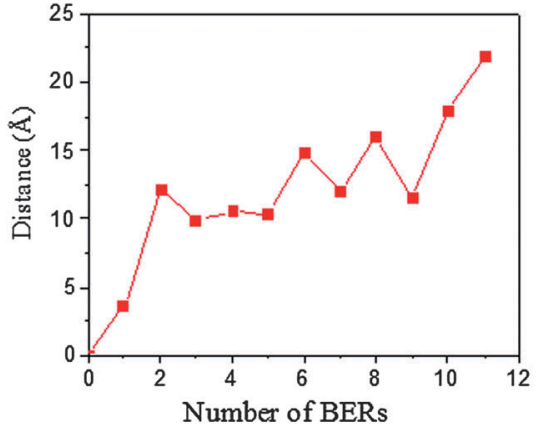

(b)

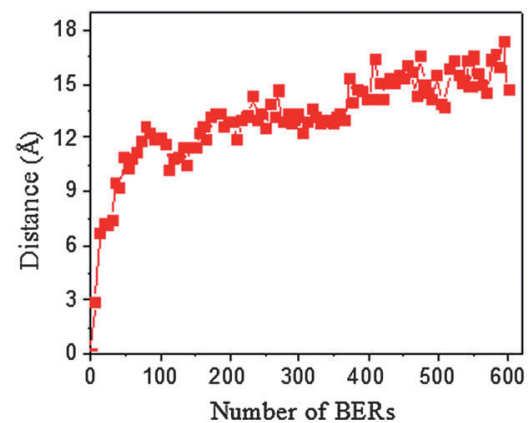

(c)

Fig. 8 (a) The trajectory and (b) the travel distance of active atoms in 11 BERs in one relay. (c) The average travel distance of active atoms as a function of number of BERs.

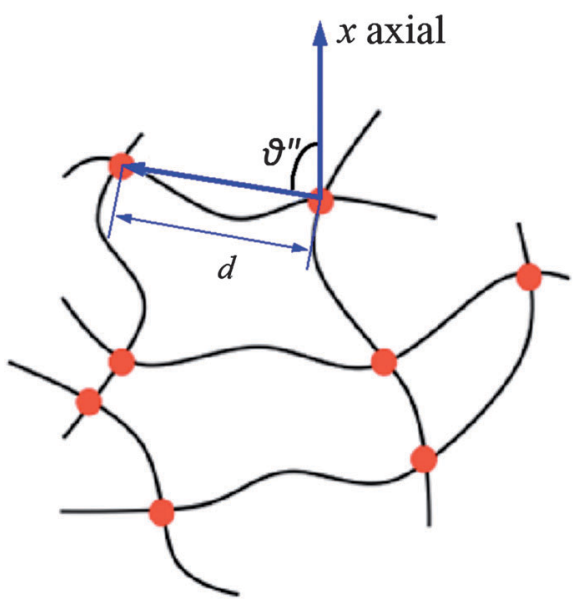

Fig. 9 The schematic graph of the distance between two neighboring crosslinking sites and the chain angle.

initial state, after 50 BER iterations and after 100 BER iterations. Student's $t$-test method is used to compare if the network topology undergoes a significant change. By comparing the distributions of the respective quantities between the initial system and the system in each BER iteration, we can obtain a two-tailed $P$ value. If the $P$ value is less than 0.05 , the two

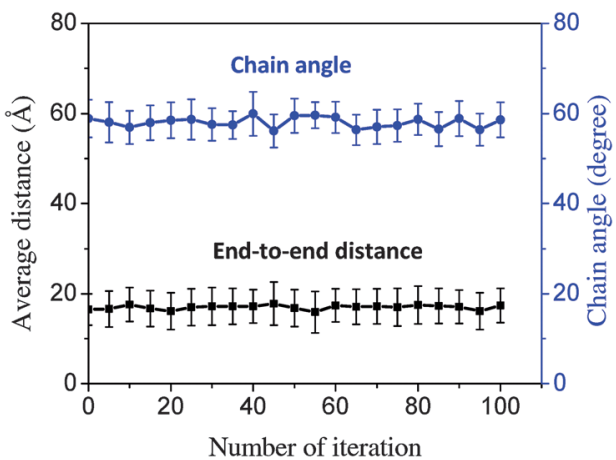

Fig. 10 The average values and their standard deviations of the distance between two crosslinking sites and the chain angle.

samples are different from each other and on the other hand, the two samples are regarded to be the same. $P$ values calculated in individual BER iteration are shown in Table 1. Since the $P$ values are greater than 0.05 , there are no significant changes in the distance between two neighboring crosslinking sites and the chain angels.

3.2.3 Mechanical properties during BER. The MD simulations of uniaxial tensile tests are carried out on the initial system and on the system after selected BER iterations at $450 \mathrm{~K}$ 
Table $1 P$ value of the distance between two neighboring crosslinking sites and the chain angle in each iteration

\begin{tabular}{|c|c|c|c|c|c|c|c|c|c|c|}
\hline Iteration & 10 & 20 & 30 & 40 & 50 & 60 & 70 & 80 & 90 & 100 \\
\hline $\begin{array}{l}P \text { value } \\
\text { Chain angle }\end{array}$ & 0.287 & 0.488 & 0.147 & 0.76 & 0.679 & 0.314 & 0.208 & 0.078 & 0.267 & 0.347 \\
\hline
\end{tabular}

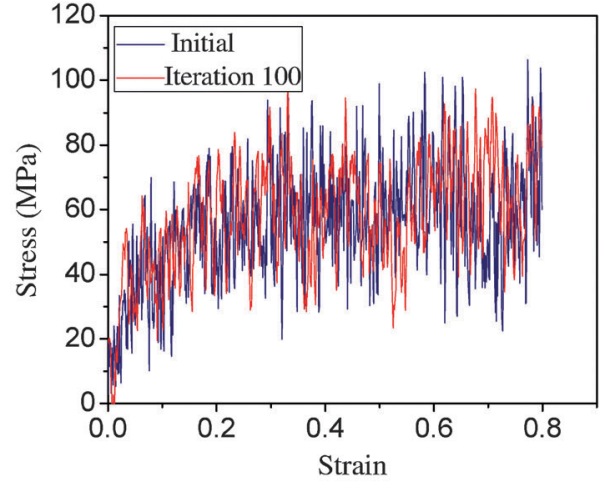

(a)

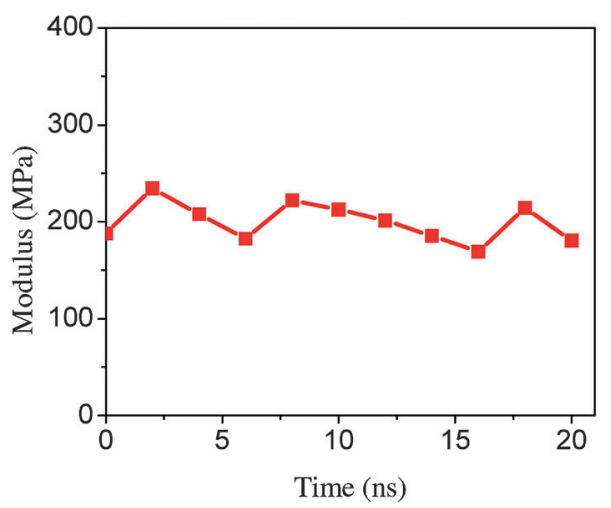

(b)

Fig. 11 (a) The stress-strain curves of the original system and the system after 100 BER iterations; (b) initial modulus as a function of time.

by continuously increasing the length of the simulation box along the $x$-direction and maintaining the volume to be constant. Fig. 11a presents the simulated stress-strain curves. In all the cases, the loading strain rate is $1 \times 10^{9} \mathrm{~s}^{-1}$ and the maximum strain is $80 \%$. The stress is determined by summing up the internal forces at individual particle point and dividing by the initial system area. The initial moduli are shown in Fig. 11b. There is only a slight variation of the initial modulus, indicating that the BERs do not change the mechanical properties of the material.

\subsection{Stress relaxation and shape reforming}

When the polymer is stretched, the network rearrangement due to BERs allows stress relaxation and shape reforming. In this section, the stress relaxation and reforming behaviors are simulated. The end-to-end distance and the chain angle are calculated during the BER process to investigate how the BER induced stress relaxation influences the network. The crosslinked structure is stretched in the $x$-direction to $80 \%$ strain at a temperature of $450 \mathrm{~K}$ with a loading rate of $1 \times 10^{9} \mathrm{~s}^{-1}$. The BER process is then performed using the method proposed above with the NVT ensemble at $450 \mathrm{~K}$. After each BER iteration, the stress in the $x$-direction is obtained, which is shown in Fig. 12a. It shows clearly that the stress relaxes as the BER process continues. Considering the time in each iteration is $200 \mathrm{ps}$, we can estimate the stress relaxation time to be $\sim 3500$ ps (see the dashed line in Fig. 12a). It should also be noted that although the stress can relax and the material behaves like a liquid when the BERs

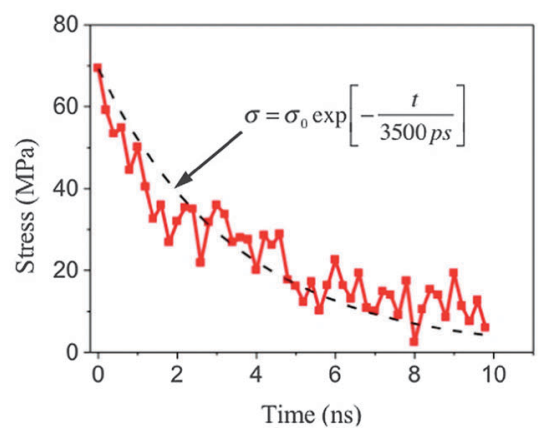

(a)

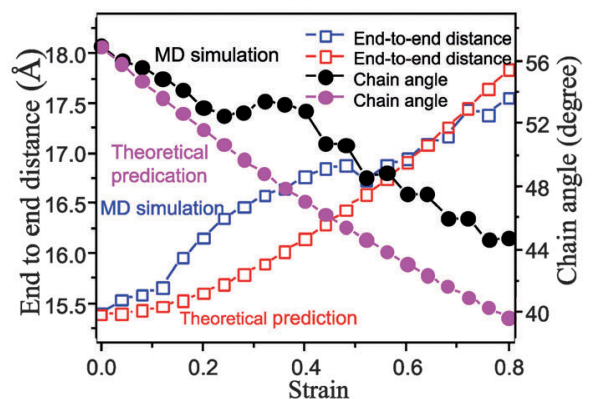

(b)

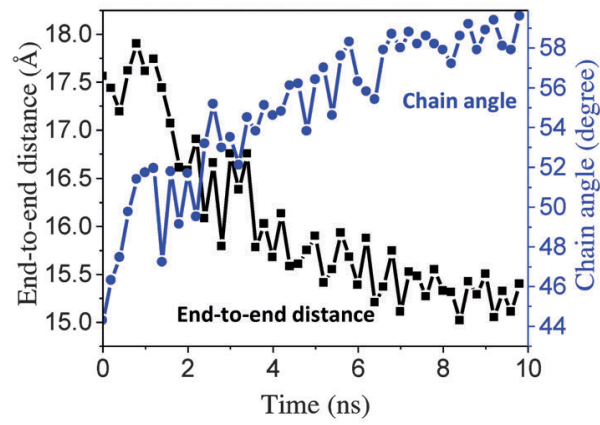

(c)

Fig. 12 (a) Stress relaxation during the BER process; evolutions of the end to end distance and the average chain angle (b) during the uniaxial tension, and (c) during BER iterations. 
are on, the network always maintains its integrity. When the BERs are slowed (such as the temperature is low and the BER kinetics becomes sluggish), the polymer behaves like a regular elastomer. If we turn off the BER, we see little stress relaxation (as shown in Fig. S4 in the ESI $\dagger$ ).

The evolutions in the end-to-end distance and the chain angle during the tension process are shown in Fig. 12b. With the deformation, the chain angle in the loading direction decreases to $\sim 44^{\circ}$, which is about a $\sim 14^{\circ}$ drop, indicating that the chains are aligned in the stretching direction. The end-to-end distance increases by about $\sim 2 \AA$. We compare the end-to-end vector in our simulation with theoretical prediction, which is a full network model of $\mathrm{Wu}$ and van de Giessen. ${ }^{48,49}$ They assumed that chains are randomly distributed. Based on this assumption, the average chain orientation under a uniaxial stretching can be calculated as

$$
\bar{\theta}=\int_{0}^{\pi / 2} \tan ^{-1}\left(\frac{1}{\lambda_{1}^{3 / 2}} \tan \theta\right) \sin \theta \mathrm{d} \theta,
$$

where $\lambda_{1}$ is the stretch and the elastomer is assumed to be incompressible. The average chain stretch can be calculated as

$$
\bar{\lambda}=\int_{0}^{\pi / 2} \sqrt{\left(\lambda_{1} \cos \theta\right)^{2}+\lambda_{1}{ }^{-1} \sin ^{2} \theta} \sin \theta \mathrm{d} \theta .
$$

Fig. 12(b) shows the comparison between the theoretical prediction and the MD simulation. It can be seen that MD simulation follows theoretical calculations.

In the BER process, Fig. 12c shows clearly that both the chain angle and the end-to-end distance recover to their original states, i.e., the chain angle increases to $\sim 59^{\circ}$, and the end-to-end distance decreases back to $15.5 \AA$ A. Both are close to their respective values in the unstretched isotropic state. These results indicate that although the tensile deformation changes the chain orientation, BER induced network rearrangement returns the network to its isotropic state.

In order to observe how different numbers of BER events cause different levels of plastic deformation, the structure after each BER iteration is allowed to recover by removing the load and switching to a NPT relaxation for $500 \mathrm{ps}$ at a temperature of $450 \mathrm{~K}$ and the atmospheric pressure. The box length in the stretched direction is measured to represent the shape change. Fig. 13a shows the initial configuration of the simulation box and Fig. 13b shows configuration after stretching. Fig. 13c presents the unloaded boxes after different numbers of BERs (50 (at $\sim 1.67 \mathrm{~ns}$ ), 100 (at $\sim 3.3 \mathrm{~ns}$ ), 200 (at $\sim 6.7 \mathrm{~ns}$ ), and 300 (at $\sim 10 \mathrm{~ns}$ ), respectively). To provide a physical insight of this process, we define the rate of BER relaxation as the number of BERs per unit time and per active group. Using this definition

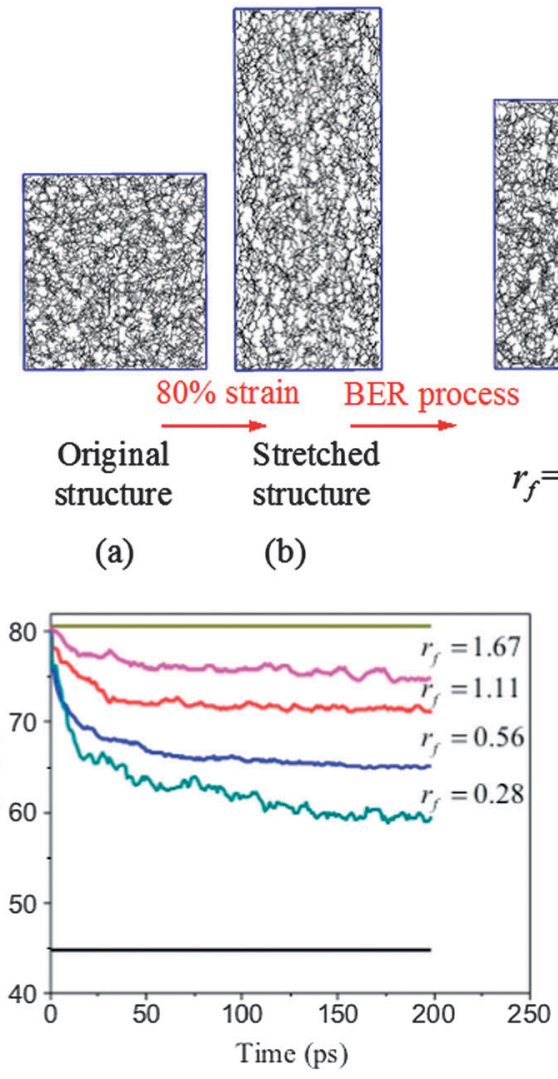

(d)

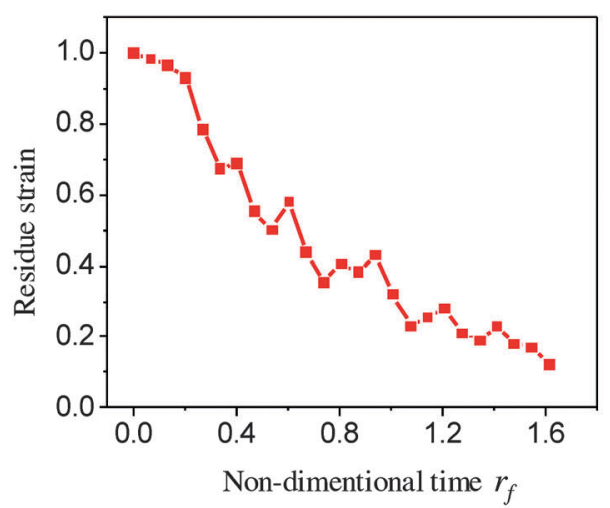

(e)

Fig. 13 BER induced plastic deformation and thermoforming. (a) The original structure. (b) The structure under 80\% strain. (c) The structures after different number of BERs. (d) The box length of the structures with different number of BERs. (e) The residual strain during the BER process. 
and the fact that we are using 180 active groups and obtain 605 BERs in $20 \mathrm{~ns}$, the BER rate is $0.168 \mathrm{~ns}^{-1}$. Therefore, the BER relaxation time is $\tau_{\mathrm{BER}}=5.95 \mathrm{~ns}$, which is close to the relaxation time measured from stress relaxation due to BERs (Fig. 12a, $3.5 \mathrm{~ns}$ ). We further normalize the times in Fig. 13c by the BER relaxation time $\tau_{\mathrm{BER}}$ as $r_{\mathrm{f}}=t / \tau_{\mathrm{BER}}$. The shape of each box thus shows the residual strain (or shape fixity) after $r_{\mathrm{f}}$ amount of BER relaxation time. Fig. 13d shows the recovery of the box during unloading at different $r_{\mathrm{f}}$. The bottom and top lines represent the original and stretched lengths of the box, respectively. The residual strain is measured and shown in Fig. 13e. The results clearly indicate that more BERs lead to better shape fixity.

\section{Discussion}

The MD simulations describe the network structure evolutions of covalent adaptable networks. It is of interest to see if the simulated network properties are consistent with the predictions from existing theories, which will verify the effectiveness of the proposed simulation method. For a network with reversible bonds, Gonzalez et al. ${ }^{50,51}$ described the diffusion and the stress relaxation by a modified reptation model. However, his relaxation time had an exponential dependence on the average number of tie points per chain due to the assumption that the chain could only make a reptation step when it was simultaneously detached from all connections. Subsequently, Leibler and coworkers ${ }^{21}$ released such a restriction by assuming that an entangled polymer chain could move by breaking a few crosslinks, which showed a faster relaxation mechanism within the network. The developed sticky reputation theory demonstrated excellent capability in predicting the dynamics of reversible networks compared with the Gonzalez's theory, which typically exhibited several orders of magnitude deviation from the experimental data, especially when the material viscous behavior was obvious. Recently, Stukhalin and coworkers ${ }^{28}$ developed a scaling theory to investigate the self-healing process in reversible networks, which was modeled by a system consisting of dangling chains with reversible stickers at one end attached to a permanently crosslinked network at the other end. They also found that the bond lifetime was the relevant time scale for the description of the stress relaxation in reversible networks. In the following discussion, the self-diffusion coefficient of the epoxy network calculated from the relaxation time is compared with those in Leibler and coworkers' theory ${ }^{21}$ and Stukhalin and coworkers' theory. ${ }^{28}$ The relationship between the bonding time and the relaxation time in the MD simulation is also compared with Stukhalin and coworkers' theory.

In Leibler et al. ${ }^{21}$ the self-diffusion coefficient is derived as:

$$
D \cong \frac{a^{2}}{2 \tau S^{2}}\left(1-\frac{9}{p}+\frac{12}{p^{2}}\right),
$$

where $a\left(a \cong b N^{1 / 2}\right)$ is the average end to end distance with $b$ denoting the monomer length and $N$ denoting the number of monomers between two crosslinking sites, $S$ is the number of stickers attached to each chain. For the epoxy network, $a=15.6 \AA, S=5 . \tau$ is the average lifetime of free stickers and $p$ is the average fraction of stickers that are closed. According to Stukalin et al. ${ }^{28} p$ can also be calculated as the fraction of lifetime of closed stickers, i.e. $p=t_{\mathrm{c}} /\left(t_{\mathrm{c}}+t_{\mathrm{o}}\right)$, where $t_{\mathrm{c}}$ is the lifetime of the closed stickers and $t_{\mathrm{o}}$ is the lifetime of the free (open) ones. The average lifetimes of free stickers and closed stickers are shown in Table 2. The initial low lifetime is due to the relatively small sampling space at the beginning of the simulation.

According to Leibler et al., ${ }^{21}$ the self-diffusion coefficient of reversible networks can be directly related to the stress relaxation time $\tau_{\mathrm{d}}$ as:

$$
D=\frac{a^{2}}{\tau_{\mathrm{d}}} \text {. }
$$

This relaxation time of the epoxy system during the MD simulation is determined to be 3500 ps from Fig. 12(a).

In Stukhalin et al. ${ }^{28}$ the dynamics of open stickers in the partner exchange regime can be described as follows:

$$
D=R_{0}^{2} / \tau_{\mathrm{b}}
$$

where $R_{0} \approx b N^{1 / 2}, D$ is the effective diffusion coefficient for partner exchange, $\tau_{\mathrm{b}}$ is the average time two stickers spent in a bonded state before a successful separation on a molecular distance.

It should be noted that in eqn (3)-(5), the average lifetime of free stickers $\tau$ and the stress relaxation time $\tau_{\mathrm{d}}$ depend on the iteration times during the MD simulation. However, due to their linear relationship with the simulation time, the comparison of the computed self-diffusion coefficients in eqn (3)-(5) is independent of the timing scale that we choose for the simulation.

The diffusion coefficients obtained from Leibler's theoretical prediction (eqn (3)), stress relaxation time (eqn (4)) and Stukhalin's theoretical prediction (eqn (5)) are plotted in Fig. 14 as a function of time. The diffusivity from stress-relaxation simulation matches very closely with Stukhalin and co-workers' prediction. The first two points in the theoretical prediction show higher values, which could be due to the smaller sampling points for the lifetime of active atoms at the beginning of the simulation. These results confirm the effectiveness of Stukhalin and coworkers' theory in studying the dynamics of a reversible covalent network.

In Stukhalin and coworkers' work, ${ }^{28}$ average lifetime of closed stickers is derived as:

$$
\tau_{\mathrm{b}} \approx \tau_{0} \exp \left(\varepsilon / k_{\mathrm{B}} T\right)
$$

\begin{tabular}{|c|c|c|c|c|c|c|c|c|c|c|}
\hline Iteration & 10 & 20 & 30 & 40 & 50 & 60 & 70 & 80 & 90 & 100 \\
\hline Average lifetime of closed stickers (ps) & 1312 & 2216 & 3128 & 3576 & 3072 & 3903 & 3798 & 3715 & 4094 & 3849 \\
\hline Average lifetime of free stickers (ps) & 1633 & 2046 & 2103 & 1967 & 2284 & 2198 & 2376 & 2388 & 2217 & 2109 \\
\hline$P$ & 0.45 & 0.52 & 0.6 & 0.65 & 0.57 & 0.64 & 0.62 & 0.61 & 0.65 & 0.65 \\
\hline
\end{tabular}

Table 2 Average lifetime of closed stickers and free stickers during the BER process, fraction of closed stickers during the BER process 


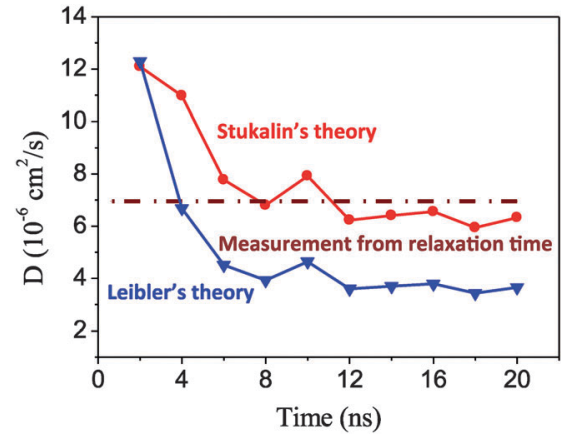

Fig. 14 Diffusion coefficients from theoretical results and relaxation time.

The average lifetime of closed stickers is equal to the time needed for a single BER. ${ }^{28}$ We assume that there are $\xi$ dangling chains in a unit volume, which means that there are $\xi$ possible exchangeable bonds. During a time interval $\mathrm{d} t$, a single exchangeable bond finishes $\frac{\mathrm{d} t}{\tau_{\mathrm{b}}}$ BERs, the total number of BERs is $\xi \frac{\mathrm{d} t}{\tau_{\mathrm{b}}}$. We also assume that after a single BER, the internal force in a single chain is totally released, the drop of stress can be related to the number of BER, which can be described as:

$$
\frac{\mathrm{d} \sigma}{\sigma}=\frac{\xi \frac{\mathrm{d} t}{\tau_{\mathrm{b}}}}{\xi}=\frac{\mathrm{d} t}{\tau_{\mathrm{b}}} .
$$

Hence,

$$
\sigma(t)=\sigma_{0} \exp \left(-\frac{t}{\tau_{\mathrm{b}}}\right),
$$

Thus we have $\tau=\tau_{\mathrm{b}}$, which means that the relaxation time is equal to the lifetime of closed stickers.

The relaxation time and the average lifetime of closed stickers during the BER process in our MD simulation are plotted in Fig. 15. The first two points of the average lifetime of the closed sticker curve show a smaller value which could be due to the small sample space. The simulation results indicate that the average lifetime of closed stickers is very close to the relaxation time of the system, which confirms the effectiveness of Stukhalin and coworkers' theory.

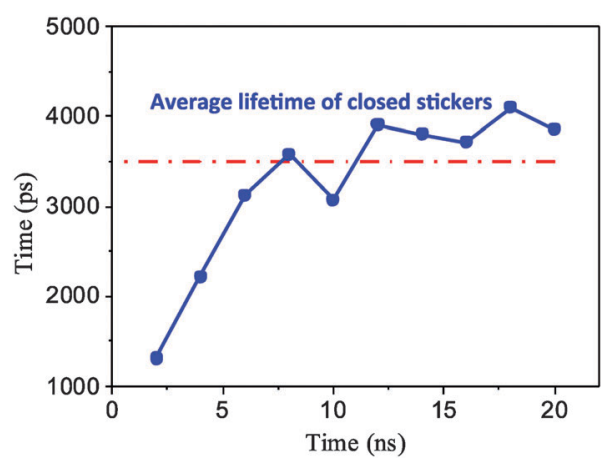

Fig. 15 The average lifetime of closed stickers and the relaxation time during the BER process.
For a network polymer that can undergo the BER process, there are two relaxation mechanisms. The first one is the conventional relaxation mechanism that is due to chain diffusion and the second one is due to BERs. Since both relaxation mechanisms can relax stress, it is important to distinguish their contributions. Here, we can obtain the relaxation time due to chain diffusion by using uniaxial tensile simulations and stress relaxation simulations where the BERs are turned off (see ESI $\dagger$ ). From these simulations, we found that the relaxation time due to chain diffusion is $\sim 2-6 \mathrm{ps}$, which is much smaller than the relaxation time due to BERs (3.5-6 ns). Therefore, in the simulations studied in this paper, the BERs are the dominant relaxation mechanism. It should be noted that in the epoxy system by Montarnal et al., ${ }^{17}$ BERs occur at temperatures significantly higher than $T_{\mathrm{g}}$, where the relaxation due to chain diffusion becomes significantly efficient (so the relaxation time is very small). As the temperature is lowered, BER rates become slower but chain diffusions are less efficient; we should see a crossover of the relaxation times. Eventually, at the temperature around $T_{\mathrm{g}}$, BER rates become almost zero and the relaxation due to chain diffusion will dominate.

It should be noted that the 90 chain system in our simulations is relatively small and may have some structure differences as compared to a larger system, especially an infinitely large network system. Such a difference and its impact on the BERs should be studied in the future. The results from this paper should be limited to a small system.

\section{Conclusions}

In this work, a detailed full atomistic molecular dynamic simulation methodology for bond exchange reactions in a covalent adaptable network is proposed. The simulations show clearly how an active group undergoes an exchange reaction, resulting in bond exchange and formation of a new active group, which subsequently undergoes additional exchange reactions. Based on the simulations, the distance between neighbored crosslinking sites, the chain angle, and the material initial modulus during the bond exchange reaction process are calculated. The results indicate that the covalent adaptable network can maintain its network integrity and mechanical properties during bond exchange reactions. Besides, the stress relaxation and thermoforming behaviors are investigated. The results indicate that although the bond exchange reactions lead to the macroscopic phenomena of stress relaxation, plastic deformation and thermoforming, the end-toend distance and the chain angle return to their original isotropic states at the macromolecular level. In addition, the comparison with the theoretical work reported by Stukhalin et al. ${ }^{28}$ confirms the effectiveness of their theory. Although the bond exchange reaction mechanism studied in this paper is limited to transesterification, this method can be extended to other covalent adaptable networks, such as Diels-Alder materials and RAFT networks, to investigate the macromolecular mechanisms. In addition, this method opens up an avenue to further research for covalent adaptable networks, such as surface welding, which is currently undergoing and will be reported in the future. 


\section{Acknowledgements}

HJQ, HY, KY and XM acknowledge the financial support from US National Science Foundation (CMMI-1404627). KY and XM also acknowledge the support from National Natural Science Foundation of China (11472207). HY acknowledges the financial support from Chinese Scholarship Council. XS acknowledges the support from NSFC (11023001). The authors also acknowledge the help and the discussions with the anonymous reviewer, whose insightful comments greatly improve the paper.

\section{References}

1 M. T. Shaw and W. J. MacKnight, Introduction to Polymer Viscoelasticity, Blackwell Science Publ, Oxford, 3rd edn, 2005, pp. 1-316.

2 C. J. Kloxin, T. F. Scott, B. J. Adzima and C. N. Bowman, Covalent Adaptable Networks (CANS): A Unique Paradigm in Cross-Linked Polymers, Macromolecules, 2010, 43(6), 2643-2653.

3 R. J. Wojtecki, M. A. Meador and S. J. Rowan, Using the dynamic bond to access macroscopically responsive structurally dynamic polymers, Nat. Mater., 2011, 10(1), 14-27.

4 C. N. Bowman and C. J. Kloxin, Covalent Adaptable Networks: Reversible Bond Structures Incorporated in Polymer Networks, Angew. Chem., Int. Ed., 2012, 51(18), 4272-4274.

5 R. Long, H. J. Qi and M. L. Dunn, Thermodynamics and mechanics of photochemcially reacting polymers, J. Mech. Phys. Solids, 2013, 61(11), 2212-2239.

6 S. J. Rowan, S. J. Cantrill, G. R. L. Cousins, J. K. M. Sanders and J. F. Stoddart, Dynamic covalent chemistry, Angew. Chem., Int. Ed., 2002, 41(6), 898-952.

7 E. B. Murphy, E. Bolanos, C. Schaffner-Hamann, F. Wudl, S. R. Nutt and M. L. Auad, Synthesis and characterization of a single-component thermally remendable polymer network: Staudinger and Stille revisited, Macromolecules, 2008, 41(14), 5203-5209.

8 S. D. Bergman and F. Wudl, Mendable polymers, J. Mater. Chem., 2008, 18(1), 41-62.

9 H. Y. Park, C. J. Kloxin, T. F. Scott and C. N. Bowman, Covalent adaptable networks as dental restorative resins: Stress relaxation by additionfragmentation chain transfer in allyl sulfidecontaining resins, Dent. Mater., 2010, 26(10), 1010-1016.

10 B. J. Adzima, C. J. Kloxin and C. N. Bowman, Externally Triggered Healing of a Thermoreversible Covalent Network via Self-Limited Hysteresis Heating, Adv. Mater., 2010, 22(25), 2784-2787.

11 T. F. Scott, A. D. Schneider, W. D. Cook and C. N. Bowman, Photoinduced plasticity in cross-linked polymers, Science, 2005, 308(5728), 1615-1617.

12 C. J. Kloxin, T. F. Scott, H. Y. Park and C. N. Bowman, Mechanophotopatterning on a Photoresponsive Elastomer, Adv. Mater., 2011, 23(17), 1977-1981.

13 K. N. Long, T. F. Scott, M. L. Dunn and H. J. Qi, Photoinduced deformation of active polymer films: Single spot irradiation, Int. J. Solids Struct., 2011, 48(14-15), 2089-2101.
14 J. Ryu, M. D’Amato, X. Cui, K. N. Long, H. J. Qi and M. L. Dunn, Photo-origami-Bending and folding polymers with light, Appl. Phys. Lett., 2012, 100(16), 161908.

15 K. Long, M. Dunn and H. Qi, Mechanics of soft active materials with phase evolution, Int. J. Plast., 2010, 26(4), 603-616.

16 X. Mu, N. Sowan, J. A. Tumbic, C. N. Bowman, P. T. Mather and H. J. Qi, Photo-Induced Bending in a Light-Activated Polymer Laminated Composite, Soft Matter, 2015, 11, 2673-2682.

17 D. Montarnal, M. Capelot, F. Tournilhac and L. Leibler, Silica-Like Malleable Materials from Permanent Organic Networks, Science, 2011, 334(6058), 965-968.

18 K. Yu, P. Taynton, W. Zhang, M. L. Dunn and H. J. Qi, Reprocessing and recycling of thermosetting polymers based on bond exchange reactions, RSC Adv., 2014, 4(20), 10108-10117.

19 Y.-X. Lu, F. Tournilhac, L. Leibler and Z. Guan, Making Insoluble Polymer Networks Malleable via Olefin Metathesis, J. Am. Chem. Soc., 2012, 134(20), 8424-8427.

20 P. Taynton, K. Yu, R. K. Shoemaker, Y. H. Jin, H. J. Qi and W. Zhang, Heat- or Water-Driven Malleability in a Highly Recyclable Covalent Network Polymer, Adv. Mater., 2014, 26(23), 3938-3942.

21 L. Leibler, M. Rubinstein and R. H. Colby, Dynamics of Reversible Networks, Macromolecules, 1991, 24(16), 4701-4707.

22 M. Rubinstein and A. N. Semenov, Thermoreversible gelation in solutions of associating polymers. 2. Linear dynamics, Macromolecules, 1998, 31(4), 1386-1397.

23 M. Rubinstein and A. N. Semenov, Dynamics of entangled solutions of associating polymers, Macromolecules, 2001, 34(4), 1058-1068.

24 A. N. Semenov and M. Rubinstein, Thermoreversible gelation in solutions of associative polymers. 1. Statics, Macromolecules, 1998, 31(4), 1373-1385.

25 K. Long, T. Scott, H. Qi, C. Bowman and M. Dunn, Photomechanics of light-activated polymers, J. Mech. Phys. Solids, 2009, 57(7), 1103-1121.

26 R. Long, H. J. Qi and M. L. Dunn, Modeling the mechanics of covalently adaptable polymer networks with temperaturedependent bond exchange reactions, Soft Matter, 2013, 9(15), 4083-4096.

27 J. Ma, X. Mu, C. N. Bowman, Y. Sun, M. L. Dunn and H. J. Qi, et al., A Photoviscoplastic Model for Photo Activated Covalent Adaptive Networks, J. Mech. Phys. Solids, 2014, 70, 84-103.

28 E. B. Stukalin, L.-H. Cai, N. A. Kumar, L. Leibler and M. Rubinstein, Self-Healing of Unentangled Polymer Networks with Reversible Bonds, Macromolecules, 2013, 46(18), 7525-7541.

29 F. Smallenburg, L. Leibler and F. Sciortino, Patchy Particle Model for Vitrimers, Phys. Rev. Lett., 2013, 111(18), 1880021.

30 D. Frenkel and B. Sumit, Understanding molecular simulation: from algorithms to applications, Elsevier, New York, 2002.

31 S. Yoshioka, Y. Aso and S. Kojima, Prediction of glass transition temperature of freeze-dried formulations by molecular dynamics simulation, Pharm. Res., 2003, 20(6), 873-878. 
32 C. Li and A. Strachan, Molecular dynamics predictions of thermal and mechanical properties of thermoset polymer EPON862/DETDA, Polymer, 2011, 52(13), 2920-2928.

33 J. H. Gou, B. Minaie, B. Wang, Z. Y. Liang and C. Zhang, Computational and experimental study of interfacial bonding of single-walled nanotube reinforced composites, Comput. Mater. Sci., 2004, 31(3-4), 225-236.

34 J. S. Bermejo and C. M. Ugarte, Chemical Crosslinking of PVA and Prediction of Material Properties by Means of Fully Atomistic MD Simulations, Macromol. Theory Simul., 2009, 18(4-5), 259-267.

35 D. R. Rottach, J. G. Curro, J. Budzien, G. S. Grest, C. Svaneborg and R. Everaers, Molecular dynamics simulations of polymer networks undergoing sequential crosslinking and scission reactions, Macromolecules, 2007, 40(1), 131-139.

36 A. Bandyopadhyay, P. K. Valavala, T. C. Clancy, K. E. Wise and G. M. Odegard, Molecular modeling of crosslinked epoxy polymers: The effect of crosslink density on thermomechanical properties, Polymer, 2011, 52(11), 2445-2452.

37 D. Montarnal, F. Tournilhac, M. Hidalgo and L. Leibler, Epoxy-Based Networks Combining Chemical and Supramolecular Hydrogen-Bonding Crosslinks, J. Polym. Sci., Part A: Polym. Chem., 2010, 48(5), 1133-1141.

38 Discovery Studio Modeling Environment: Release 5.5 [press release], San Diego, 2007.

39 H. Sun, Ab-Initio Calculations And Force-Field Development For Computer-Simulation Of Polysilanes, Macromolecules, 1995, 28(3), 701-712.

40 S. Plimpton, Fast Parallel Algorithms For ShortRange Molecular-Dynamics, J. Comput. Phys., 1995, 117(1), 1-19.
41 S. Nose, A Unified Formulation Of The Constant Temperature Molecular-Dynamics Methods, J. Chem. Phys., 1984, 81(1), 511-519.

42 W. G. Hoover, Canonical Dynamics - Equilibrium PhaseSpace Distributions, Phys. Rev. A: At., Mol., Opt. Phys., 1985, 31(3), 1695-1697.

$43 \mathrm{C} . \mathrm{Wu}$ and $\mathrm{W}$. Xu, Atomistic molecular modelling of crosslinked epoxy resin, Polymer, 2006, 47(16), 6004-6009.

44 V. Varshney, S. S. Patnaik, A. K. Roy and B. L. Farmer, A molecular dynamics study of epoxy-based networks: Crosslinking procedure and prediction of molecular and material properties, Macromolecules, 2008, 41(18), 6837-6842.

45 D. R. Heine, G. S. Grest, C. D. Lorenz, M. Tsige and M. J. Stevens, Atomistic simulations of end-linked poly(dimethylsiloxane) networks: Structure and relaxation, Macromolecules, 2004, 37(10), 3857-3864.

46 X. X. Chen, M. A. Dam, K. Ono, A. Mal, H. B. Shen and S. R. Nutt, et al., A thermally re-mendable cross-linked polymeric material, Science, 2002, 295(5560), 1698-1702.

47 G. Moad, E. Rizzardo and S. H. Thang, Radical additionfragmentation chemistry in polymer synthesis, Polymer, 2008, 49(5), 1079-1131.

$48 \mathrm{P}$. Wu and E. Van der Giessen, On improved network models for rubber elasticity and their applications to orientation hardening in glassy polymers, J. Mech. Phys. Solids, 1993, 41(3), 427-456.

49 P. Wu and E. Van Der Giessen, On improved 3-D nonGaussian network models for rubber elasticity, Mech. Res. Commun., 1992, 19(5), 427-433.

50 A. E. Gonzalez, Viscosity of Ionomer Gels, Polymer, 1983, 24(1), 77-80.

51 A. E. Gonzalez, Viscoelasticity of Ionomer Gels. 2. The Elastic-Moduli, Polymer, 1984, 25(10), 1469-1474. 